\title{
THỰC TRẠNG PHÁT ÂM CÁC ÂM XÁT /s/ VÀ / / / TIẾNG ANH CỦA SINH VIÊN CHUYÊN NGÀNH NGÔN NGŨ̉ ANH TRƯỜ'G ĐẠI HỌC CÔNG NGHIỆP TP. HỒ CHÍ MINH
}

\author{
NGUYẼ̃N THU HÀ, PHAN THANH HUYỀN, NGUYẼ̃N ĐÌNH LUẬT \\ Truờng Đại học Công nghiệp Thành phố Hồ Chí Minh; \\ hathu41@gmail.com, phanthanhhuyen@iuh.edu.vn,luatmh@gmail.com
}

Tóm tắt: Tiếng Anh hiện nay là ngôn ngữ toàn cầu. Ý thức được điều này, các trường học và sinh viên, học sinh Việt Nam đầu tư cho việc dạy và học tiếng Anh rất nhiều. Tuy nhiên một thực trạng đáng báo động là dù thực sự đầu tư nhưng sinh viên học sinh Việt Nam thường xuyên gặp tình huống nói tiếng Anh người bản ngữ không hiểu và nghe người bản ngữ nói họ không hiểu... Bài nghiên cứu khảo sát 200 sinh viên chuyên ngành Ngôn ngữ Anh trường đại học Công nghiệp TP. Hò Chí Minh - những nhà giáo, phiên dịch viên tiếng Anh tương lai, thống kê thực trạng phát âm trong giới hạn phát âm âm xát tiếng Anh một loại âm hay bị phát âm sai để lý giải hiện tượng trên và đề xuất giải pháp khắc phục lỗi phát âm, cải thiện kỹ năng nói - một kỹ năng quan trọng trong việc sử dụng tiếng Anh để góp phần tích cực cho hoạt động nghề nghiệp của sinh viên sau khi tốt nghiệp và đáp ứng nhu cầu xã hội.

Từ khóa: lỗi, âm, âm tiết, âm xát, phát âm, Ngữ âm thực hành và Ngữ âm-Ầm vị học

\section{AN INVESTIGATION INTO IUH ENGLISH MAJORS' CAPACITY TO ARTICULATE ENGLISH FRICATIVES /s/ AND / / /}

\begin{abstract}
English is now a global language. Being aware of this, Vietnamese schools and students invest heavily in teaching and learning English. However, it is an undeniable fact that despite significant investment, Vietnamese students still struggle to utter English words and thus frequently encounter situations in which native speakers do not understand what they say or vice versa. Hence, it is of great importance to uncover the reasons behind this incompetence. As informants for the investigation, 200 IUH English-majored students, future English teachers and interpreters, were randomly and conveniently sampled for a pronunciation test centering on two English fricative /s/ and $/ \mathrm{J} /$, which are seemingly mispronounced on a frequent basis. The investigation was conducted with the aim of finding out the explanation for the phenomenon and propose solutions to partially improve the students' pronunciation, specifically that of English fricatives /s/ and $/ / /$, which hopefully will, in turn, contributes positively to the professional activities of those students after graduation.
\end{abstract}

Key words. errors, sounds, syllables, fricatives, pronunciation, Applied Phonetics, Phonetics-Phonology.

\section{1 ĐẶT VẤN ĐỀ}

Trong xu thế toàn cầu hóa ngày nay, tầm quan trọng của tiếng Anh là không thể phủ nhận vì nó được dùng phổ biến ở mọi nơi trên thế giới. Tiếng Anh phát triển cùng với sự phát triển của công nghệ, y học, kỹ thuật và giáo dục... Ở Việt Nam, sinh viên nhận thức được tầm quan trọng của tiếng Anh vì họ có thể tìm được một công việc chất lượng cao, giao tiếp được với thế giới bên ngoài, tiếp cận với nguồn tài liệu khoa học tiển tiến... Các trường đại học, cao đẳng hiện nay tiến hành giảng dạy tiếng Anh và nhiều nội dung bằng tiếng Anh cũng như quy định chuẩn đầu ra ngoại ngữ (phổ biến là tiếng Anh) cho sinh viên tốt nghiệp ra trường. Tuy nhiên, theo bài Thị trường lao động Asean lắm co hội, nhiều thách thức đăng trên báo Pháp Luật số ra ngày 01/01/2016, “....vấn đề đáng lo ngại nhất đối với sinh viên Việt Nam là năng lực ngoại ngữ như tiếng Anh còn nhiều hạn chế". Điều đáng quan tâm là nhiều sinh viên tốt nghiệp ra trường không thể nói tiếng Anh lưu loát, hoặc có vẻ trôi chảy nhưng người nước ngoài nghe lại rất khó hiểu. Một trường hợp phổ biến khác là các em nghe những từ cơ bản đã biết nhưng không thể nhận ra những từ đó khi người khác phát âm. Vì sao lại có hiện tượng này? Câu trả lời là thực tế dạy và học tiếng 
Anh ở trường phổ thông chú trọng quá nhiều vào ngữ pháp và đọc hiểu mà bỏ qua hai kỹ năng quan trọng là nghe và nói dẫn đến đa số học sinh phát âm sai và khi nghe từ đó được phát âm khác thì không hiểu.

Đề tài nghiên cứu về phát âm tiếng Anh cho đến thời điểm này, có một số tác giả như Nguyễn Thanh Tâm, giảng viên Khoa Ngôn ngữ \& VHQT, Trường Đại học Văn hóa Hà Nội với bài viết đăng năm 2014 "Lỗi phát âm tiếng Anh thường gặp của sinh viên không chuyên, nguyên nhân và biện pháp khắc phục", Đinh Thị Phương Hoa, giảng viên trường Đại học Sư phạm Nghệ thuật Trung ương với nghiên cứu được đăng năm 2014 "Một số giải pháp tăng cường các kỹ năng thực hành tiếng Anh cho sinh viên không chuyên ngữ Trường Đại học Sư phạm Nghệ thuật Trung ương", "Một số giải pháp cải thiện kỹ năng nói tiếng Anh cho sinh viên khối nghệ thuật"; Lê Minh Thu, khoa Sư phạm Tiếng Anh - ĐH Ngoại Ngữ ĐHQGHN với đề tài công bố năm 2016 "Việc áp dụng phương pháp tự quay bài nói trong việc cải thiện phát âm của học viên langmaster trình độ cơ bản",... nhưng chưa có một tác giả hay một đề tài nào nghiên cứu chuyên sâu về thực trạng phát âm tiếng Anh, lỗi phát âm tiếng Anh của sinh viên chuyên ngành Ngôn ngữ Anh và những âm hay phát âm sai, nhất là âm xát/s/ và $/ \delta /$. Đây là các phụ âm mà trong quá trình giảng dạy chúng tôi nhận thấy sinh viên phát âm sai rất nhiều. Vì vậy, nhóm nghiên cứu muốn đi sâu tìm hiểu thực trạng phát âm âm xát $/ \mathrm{s} /$ và $/ \int /$ tiếng Anh của sinh viên chuyên ngành ngôn ngữ Anh trường Đại học Công nghiệp TP. Hồ Chí Minh, từ đó đề xuất các giải pháp khắc phục trong phương pháp giảng dạy của giáo viên và học tập của sinh viên góp phần nâng cao chất lượng đào tạo của trường.

Bài nghiên cứu được thực hiện dựa trên những câu hỏi nghiên cứu như sau:

- Sinh viên có phát âm sai âm xát /s/ và / $/$ / như dự đoán của tác giả và ý kiến bộ môn không?

- Lỗi sai khi phát âm các phụ âm nói trên có phổ biến không?

- Nhóm sinh viên nào có số lỗi phát âm nhiều hơn? Lỗi sai phổ biến ở âm nào? Vị trí nào ? Trong từ riêng lẻ hay trong câu? Các lỗi đó có tuân theo qui luật nào không/có tính hệ thống hay không khi đối chiếu kết quả của hai nhóm sinh viên?)

\section{PHƯƠNG PHÁP NGHIÊN CÚU}

Để thực hiện đề tài, nhóm chúng tôi đã sử dụng những phương pháp nghiên cứu khác nhau:

Phương pháp điều tra bằng các bảng câu hỏi tìm hiểu về yếu tố vùng miền, quá trình học, cách thức học, phương pháp học, thói quen học, khó khăn khi học tiếng Anh của sinh viên; bài kiểm tra phát âm trong từ riêng lẻ với một âm xát ở các vị trí âm đầu, âm giữa, âm cuối, từ riêng lẻ có cả hai âm xát, hai âm xát trong cụm từ ở các vị trí khác nhau, hai âm xát ở các vị trí khác nhau trong câu.

Phương pháp thực nghiệm, ghi âm trực tiếp từng sinh viên và lắng nghe xác định lỗi phát âm sai của sinh viên.

Để đảm bảo tính khách quan và độ tin cậy của kết quả phận định đúng hay sai trong việc phát âm các phụ âm trên, phần ghi âm của các sinh viên tham gia khảo sát được giao cho hai giáo viên bản xứ chấm theo các phiếu đã được lập ra. Mỗi giáo viên chấm 50 đoạn ghi âm của sinh viên năm thứ nhất và 50 đoạn ghi âm của sinh viên năm thứ ba.

Sau khi mã hóa và nhập dữ liệu vào Excel, chúng tôi sử dụng chức năng lọc thông tin của phần mềm này để tìm kiếm các từ có chứa những nội dung cần khảo sát, sau đó thống kê tần suất để phân tích, so sánh và đối chiếu thông tin để tìm ra các quy luật có thể tồn tại về lỗi phát âm của hai nhóm sinh viên.

Ngoài ra để tìm hiểu mối tương quan giữa khả năng phát âm của sinh viên và các yếu tố có thể có tác động đến quá trình học phát âm của sinh viên, dữ liệu từ bảng khảo sát thông tin của sinh viên cũng được mã hóa và đối chiếu.

\section{KẾT QUẢ NGHIÊN CỨU}

\subsection{Kết quả khảo sát}

Phần thu âm của hai nhóm sinh viên cho thấy một điều khá hợp lí là sinh viên năm nhất phát âm sai nhiều hơn sinh viên năm 3 trong hầu hết các nội dung, cụ thể là khi phát âm âm xát $/ \mathrm{s} /$ trong từ riêng lẻ, số lượt phát âm sai của sinh viên năm nhất chiểm khoảng $25 \%$ so với mức $7 \%$ của sinh viên năm 3 , và tỉ lệ phát âm sai âm xát $/ \mathrm{J} /$ là gần $14 \%$ đối với sinh viên năm nhất và $10 \%$ đối với sinh viên năm ba. Tương tự, khi các âm tiết có chứa hai phụ âm nói trên được sắp xếp xen kẽ và không có dấu hiệu rõ ràng âm nào 
đang được kiểm tra, $32 \%$ số lượt đọc sai là của sinh viên năm nhất và $27,5 \%$ thuộc về sinh viên năm thứ ba.

Điều đáng ngạc nhiên duy nhất là khi đọc các âm tiết có chứa âm xát /s/ và / // trong một câu, sự khác biệt giữa hai nhóm không đáng kể, thậm chí số lượt phát âm đúng của nhóm sinh viên năm nhất lại nhiều hơn nhóm sinh viên năm ba khoảng $1,3 \%$. Điều đó cho thấy sinh viên năm thứ ba dù đã học các môn Ngữ âm thực hành và Ngữ âm-Âm vị học nhưng vẫn còn gặp khó khăn khi phát âm các âm xát đang được xem xét trong môi trường có nhiều từ xung quanh.

Nhìn chung, sinh viên năm nhất phát âm sai khá nhiều các âm tiết có chứa âm xát /s/ (xấp xỉ 25\%). Sinh viên hai nhóm gặp nhiều khó khăn hơn khi phát âm các âm tiết và câu tổng hợp hai phụ âm nêu trên (tỉ lệ đọc sai của sinh viên năm nhất ở cấp độ câu là $31,8 \%$ và năm ba là $33,2 \%$ ). Đây cũng vấn đề sẽ được xem xét chi tiết hơn trong các phần tiếp theo.

Bảng 1 . Kết quả thu âm tổng quát của sinh viên năm 1 và năm 3

\begin{tabular}{|c|c|c|c|c|c|c|c|c|c|c|c|c|c|c|c|c|}
\hline & \multicolumn{8}{|c|}{ Từ riêng lẻ } & \multirow{2}{*}{\multicolumn{4}{|c|}{$\begin{array}{l}\text { Tổng hợp cả } 2 \text { âm } \\
\text { Trong từ }\end{array}$}} & \multirow{2}{*}{\multicolumn{4}{|c|}{$\begin{array}{c}\text { Tổng hợp cả } 2 \text { âm Trong } \\
\text { câu }\end{array}$}} \\
\hline & \multicolumn{4}{|c|}{ /s/ } & \multicolumn{4}{|c|}{ Igl } & & & & & & & & \\
\hline & \multicolumn{2}{|c|}{$\begin{array}{l}\text { Phát âm } \\
\text { đúng }\end{array}$} & \multicolumn{2}{|c|}{ Phát âm sai } & \multicolumn{2}{|c|}{$\begin{array}{l}\text { Phát âm } \\
\text { đúng }\end{array}$} & \multicolumn{2}{|c|}{ Phát âm sai } & \multicolumn{2}{|c|}{$\begin{array}{l}\text { Phát âm } \\
\text { đúng }\end{array}$} & \multicolumn{2}{|c|}{ Phát âm sai } & \multicolumn{2}{|c|}{$\begin{array}{l}\text { Phát âm } \\
\text { đúng }\end{array}$} & \multicolumn{2}{|c|}{ Phát âm sai } \\
\hline & số lượt & $\begin{array}{l}\text { tì lê } \\
(\%)\end{array}$ & $\begin{array}{l}\text { số } \\
\text { lượt }\end{array}$ & $\begin{array}{l}\text { tỉ lẹ } \\
(\%)\end{array}$ & số lượt & $\begin{array}{l}\text { tì lê } \\
(\%)\end{array}$ & $\begin{array}{l}\text { số } \\
\text { lượt }\end{array}$ & $\begin{array}{l}\text { ti lẹ } \\
(\%)\end{array}$ & $\begin{array}{c}\text { số } \\
\text { lượt }\end{array}$ & $\begin{array}{l}\text { tì lê } \\
(\%)\end{array}$ & $\begin{array}{l}\text { số } \\
\text { lượt }\end{array}$ & $\begin{array}{l}\text { ti lê } \\
(\%)\end{array}$ & số lượt & $\begin{array}{l}\text { tì lê } \\
(\%)\end{array}$ & $\begin{array}{c}\text { số } \\
\text { lượt }\end{array}$ & $\begin{array}{l}\text { tì lê } \\
(\%)\end{array}$ \\
\hline Năm 1 & 1652 & 75.1 & 548 & 24.9 & 1728 & 86.4 & 272 & 13.6 & 544 & 68.0 & 256 & 32.0 & 1364 & 68.2 & 636 & 31.8 \\
\hline Năm 3 & 2044 & 92.9 & 156 & 7.1 & 1801 & 90.1 & 199 & 10.0 & 580 & 72.5 & 220 & 27.5 & 1337 & 66.9 & 663 & 33.2 \\
\hline
\end{tabular}

\subsection{Kết quả chi tiết}

Kết quả thu được về số lần phát âm đúng và sai của sinh viên hai nhóm khi phát âm các âm tiết riêng lẻ có chứa hai phụ âm xát $/ \mathrm{s} /$ và $/ \mathrm{g} /$ được tổng hợp trong bảng 2 dưới đây. Để tìm hiểu chi tiết hơn về các cơ chế mắc lỗi, những khó khăn mà sinh viên hai nhóm mắc phải, cũng như tìm hiểu xem có mối tương quan nào mang tính hệ thống của các lỗi sai của sinh viên hai nhóm hay không, bài viết sẽ tiếp tục đi sâu so sánh kết quả thu âm của hai nhóm sinh viên khi phát âm âm tiết riêng lẻ, với từng phụ âm xát /s/ và / $\int /$ khi các âm này được phân bố ở các vị khác nhau trong một âm tiết.

Bảng 2. Kết quả thu âm xét theo vị trí của hai âm của sinh viên năm 1 và năm 3

\begin{tabular}{|c|c|c|c|c|c|c|c|c|c|c|}
\hline \multirow{10}{*}{$\begin{array}{c}\text { Từ } \\
\text { riêng } \\
\text { lẻ }\end{array}$} & \multirow{4}{*}{$\begin{array}{l}\text { Phụ } \\
\text { âm }\end{array}$} & \multirow{4}{*}{ Vị trí âm } & \multicolumn{8}{|c|}{ Nhóm sinh viên } \\
\hline & & & \multicolumn{4}{|c|}{ Năm 1} & \multicolumn{4}{|c|}{ Năm 3} \\
\hline & & & \multicolumn{2}{|c|}{ Phát âm đúng } & \multicolumn{2}{|c|}{ Phát âm sai } & \multicolumn{2}{|c|}{ Phát âm đúng } & \multicolumn{2}{|c|}{ Phát âm sai } \\
\hline & & & số lượt & tỉ lệ (\%) & số lượt & tỉ lệ (\%) & số lượt & tỉ lệ (\%) & số lượt & tỉ lệ (\%) \\
\hline & \multirow{3}{*}{$/ \mathrm{s} /$} & đầu & 598 & 74,75 & 202 & 25,25 & 736 & 92 & 64 & 8 \\
\hline & & giữa & 422 & 70,3 & 178 & 29,6 & 546 & 91 & 54 & 9 \\
\hline & & cuối & 632 & 79 & 168 & 21 & 762 & 95.25 & 38 & 4.75 \\
\hline & \multirow{3}{*}{$/ \mathrm{J} /$} & đầu & 652 & 93.1 & 48 & 6.9 & 664 & 94,9 & 36 & 5,1 \\
\hline & & giữa & 462 & 77 & 138 & 23 & 567 & 94,5 & 33 & 5,5 \\
\hline & & cuối & 614 & 87.7 & 86 & 12.3 & 570 & 81,4 & 130 & 18,6 \\
\hline
\end{tabular}

\subsection{So sánh kết quả âm xát /s/ trong từ độc lập ở các vị trí khác nhau giữa hai nhóm sinh viên}

Biểu đồ cho thấy số lượt phát âm sai ở hai nhóm sinh viên cho thấy có sự nhất quán đáng kể, vì số lượt phát âm sai chiếm tỉ lệ cao nhất khi âm xát/s/ nằm ở giữa âm tiết, đối với sinh viên năm nhất là xấp xỉ $30 \%$ và sinh viên năm thứ ba là $9 \%$. Vị trí phân bố gây khó khăn xếp thứ hai là khi âm xát /s/ nằm ở đầu một âm tiết, tuy nhiên mức độ sai của sinh viên năm nhất đáng kể hơn nhiều, với tỉ lệ đọc sai là trên 
$25 \%$, so với $8 \%$ ở sinh viên năm ba. Vị trí cuối âm tiết có lẽ là vị trí ít thách thức nhất đối với cả hai nhóm sinh viên, lỗi sai ở sinh viên năm nhất là $21 \%$ và chỉ xấp xỉ $5 \%$ ở sinh viên năm ba.

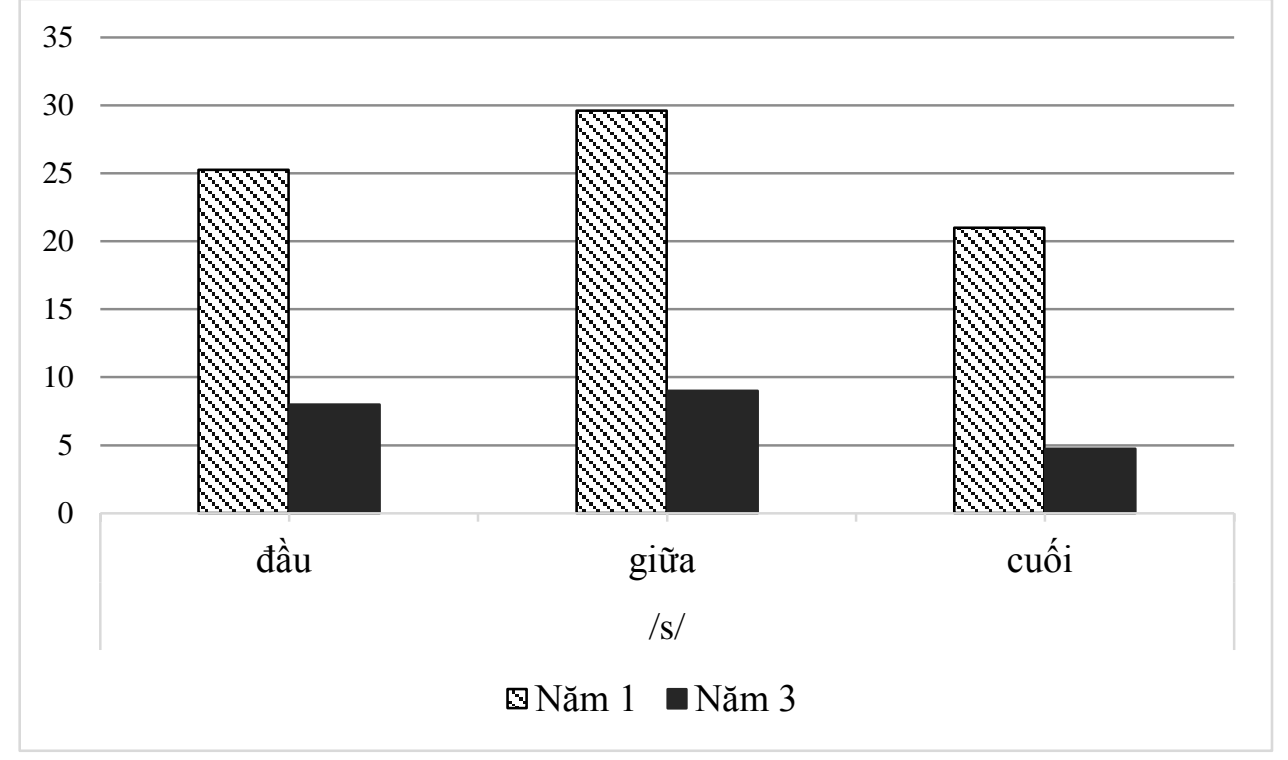

Bảng 3. Lỗi phát âm âm /s/ trong từ độc lập của sinh viên năm 1 và năm 3

3.4 So sánh kết quả âm / J/ trong từ độc lập ở các vị trí khác nhau giữa hai nhóm sinh viên

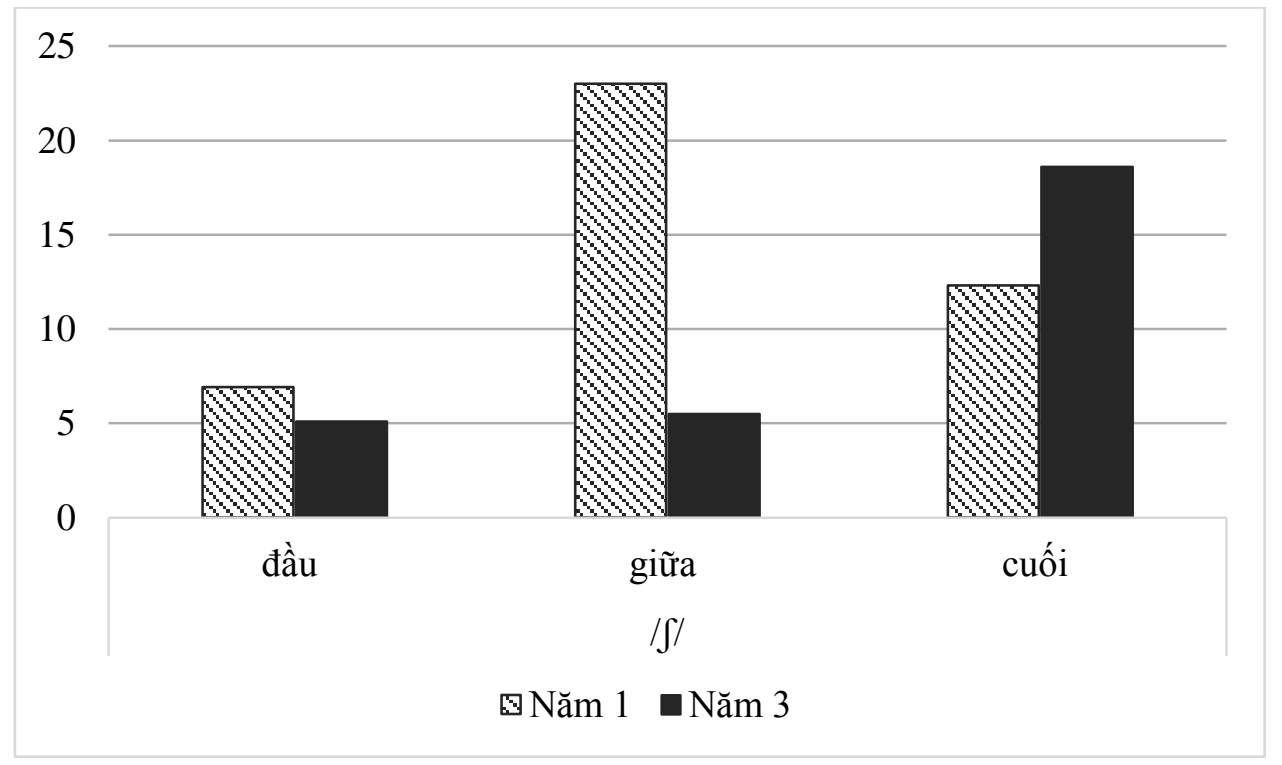

Bảng 4. Lỗi phát âm âm /S/ trong từ độc lập của sinh viên năm 1 và năm 3

Xét về lỗi sai khi phát âm âm xát /J/ ở các vị trí khác nhau trong âm tiết riêng lẻ, biểu đồ trên cho thấy sinh viên phát âm sai nhiều nhất khi âm xát / / nằm ở vị trí giữa âm tiết, với tỉ lệ phát âm sai chiếm $23 \%$, còn sinh viên năm ba thì gặp khó khăn nhiều nhất khi âm xát / $/$ / được phân bố cuối âm tiết, với số lượt phát âm sai xấp xỉ $20 \%$. Vị trí cuối âm tiết của âm xát / / / cũng gây khó khăn nhiều hơn cho sinh viên năm nhất so với vị trí đầu âm tiết, có số lượt phát âm sai chiếm tỉ lệ khoảng $12 \%$. Thế nhưng đối với sinh viên năm ba, vị trí cuối và giữa âm tiết của âm xát /J/ không gây nhiều khó khăn, và tỉ lệ phát âm sai ở hai vị trí này khá tương đồng, xấp xỉ $5 \%$.

\subsection{So sánh kết quả phát âm hai âm xát /s/ và // / trong các âm tiết có chứa cả hai âm và trong câu giữa hai nhóm sinh viên}


Như đã nêu trong bảng tổng hợp kết quả, hai nhóm sinh viên phát âm sai nhiều nhất khi phát âm các âm tiết có chứa cả hai phụ âm hoặc các âm tiết có chứa một trong hai âm được trộn lẫn vào nhau theo nhóm và theo từng câu. Theo thống kê từ bảng tóm tắt dưới đây, số lượt phát âm sai của sinh viên năm nhất khi phát âm xát /s/ và / $/$ trong cả hai trường hợp đều xấp xỉ $32 \%$. Số lượt phát âm sai của sinh viên năm ba khi phát âm $/ \mathrm{s} /$ trong các âm tiết tổng hợp chiếm tương đương $28 \%$ và trong câu là khoảng $33 \%$ đây cũng là trường hợp duy nhất lỗi sai của sinh viên năm ba chiếm tỉ lệ cao hơn so với sinh viên năm nhất.

Khi phát âm các âm tiết có chứa âm xát /s/ và / / / trong dãy âm tiết tổng hợp và trong câu, sinh viên không có bất kì dấu hiệu nào từ đề bài kiểm tra và phải tự nhận diện từ nào hoặc âm tiết nào có chứa âm xát /s/ và âm / / / để đọc. Lúc này lỗi sai của sinh viên có thể do hai nguyên nhân như sau. Thứ nhất, các sinh viên không kiểm soát được các bộ phận tham gia vào quá trình cấu âm, nói cách khác là vị trí cấu âm khi phải liên tục chuyển đổi các vị trí cấu âm hay luân phiên sử dụng các bộ phận cấu âm khác nhau. Thứ hai, có thể các sinh viên nhầm lẫn giữa chữ viết, thậm chí là chữ viết tiếng Việt với phụ âm được sử dụng, dẫn đến việc áp dụng sai nguyên tắc phát âm của tiếng Anh hoặc sử dụng nguyên tắc phát âm của tiếng Việt dựa trên chữ viết vào việc phát âm các từ và âm tiết tiếng Anh. Vì vậy bài viết tiếp tục đi sâu xử lí và phân tích dữ liệu phát âm của sinh viên liên quan đến lỗi sai khi xét về vị trí của các phụ âm đang xem xét và cách thức các em mắc lỗi (thay thế âm, lược bỏ âm, nhầm lẫn chữ viết với phụ âm) để tìm hiểu liệu các lỗi sai đó có tuân theo quy luật cụ thể nào, nhất là các qui luật có liên quan đển sự khác nhau về hình thái ngôn ngữ giữa tiếng Việt và tiếng Anh hay không.

Bảng 5. Lỗi phát âm âm/s/ và $/ / /$ trong từ có chứa cả hai âm và trong câu của sinh viên năm 1 và năm 3

\begin{tabular}{|c|c|c|c|c|c|c|c|c|}
\hline & \multicolumn{4}{|c|}{ Tổng hộp cả 2 âm trong từ } & \multicolumn{4}{|c|}{ Tổng hợp cả 2 âm trong câu } \\
\hline & \multicolumn{2}{|c|}{ Phát âm đúng } & \multicolumn{2}{|c|}{ Phát âm sai } & \multicolumn{2}{|c|}{ Phát âm đúng } & \multicolumn{2}{|c|}{ Phát âm sai } \\
\hline & số lượt & tỉ lẹ (\%) & số lượt & tỉ lệ (\%) & số lượt & tỉ lẹ (\%) & số lượt & tỉ lẹ $(\%)$ \\
\hline Năm 1 & 544 & 68.0 & 256 & 32.0 & 1364 & 68.2 & 636 & 31.8 \\
\hline Năm 3 & 580 & 72.5 & 220 & 27.5 & 1337 & 66.9 & 663 & 33.2 \\
\hline
\end{tabular}

\section{KẾT LUẬN}

Từ kết quả khảo sát trong các bảng 1, 2, 3 cho thấy sinh viên phát âm sai trong tất cả các nội dung, năm nhất phát âm sai nhiều hơn sinh viên năm ba và hai nhóm sinh viên phát âm sai nhiều nhất khi phát âm các âm tiết có chứa cả hai phụ âm xát hoặc các âm tiết có chứa một trong hai phụ âm xát được trộn lẫn vào nhau theo nhóm và theo từng câu là $28 \%$ đến $33 \%$. Đối với sinh viên chuyên ngành $\mathrm{Ngôn}$ ngữ Anh thì tỉ lệ các từ các em phát âm sai như vậy là khá cao. Để giúp sinh viên cải thiện kỹ năng phát âm, chúng tôi đề xuất một số vấn đề như sau:

Về phía nhà trường cần đầu tư xây dựng cơ sở vật chất cho phương thức dạy và học E-Learning, phòng Multimedia Lab nhiều tính năng hữu ích cho việc dạy của giáo viên cũng như việc học của sinh viên khoa Ngoại ngữ để cải thiện khả năng phát âm, kỹ năng nói tiếng Anh. Các phòng học này có thể được trang bị các phần mềm thể hiện rõ khẩu hình của người bản xứ khi phát âm để sinh viên có thể tham khảo và luyện tập theo.

Về phía giảng viên, cần xác định rằng sinh viên đã ở độ tuổi trưởng thành nên khả năng học phát âm tiếng nước ngoài chắc chắn sẽ bị ảnh hưởng sâu sắc bởi tiếng mẹ đẻ. Chính vì vậy, giảng viên không thể chỉ dựa vào khả năng nghe và bắt chước của các em để giảng dạy. Về mặt lý thuyết, giảng viên cần mô tả chi tiết và đối chiếu phương thức cấu âm của các phụ âm xát /s/ và / $/$ /, có thể kết hợp so sánh với các phụ âm khác có phương thức cấu âm gần giống như âm $/ \mathrm{z} /$ và âm $/ 3 /$, chú trọng đến các nét khu biệt của mỗi âm. Ngoài ra giảng viên còn có thể so sánh hai âm xát nói trên với các phụ âm có nét tương đồng trong tiếng Việt như âm /s/ và /ṣ/. Về mặt thực hành, khi giảng dạy âm $/ \mathrm{s} /$, giảng viên cần chú ý cho sinh viên luyện tập nhiều và thường xuyên phát âm này ở cả ba vị trí đầu, giữa và cuối từ, đối với âm $/ \mathrm{S} / \mathrm{cần} \mathrm{lưu} \mathrm{ý}$ vị trí giữa và cuối từ. Một phần không thể thiếu nữa là luyện tập phát âm các âm này trong câu, vốn luôn là phần phát âm thực tế nhât và cũng khó nhất mà sinh viên luôn mắc lỗi nhiều nhất. Trên đây là những vị trí sinh viên thường đọc sai mà giảng cần lưu ý để nâng cao hiệu quả giảng dạy. 
Về phía sinh viên cần tự ý thức được việc học tiếng Anh trước hết là phải luyện phát âm đúng bằng cách tìm hiểu phương thức cấu âm, nắm vững vị trí cấu âm các phụ âm xát $/ \mathrm{s} /, / \mathrm{J} /$, so sánh, tìm ra sự khác biệt giữa phụ âm xát $/ \mathrm{s} /, / \mathrm{s} /$ tiếng Việt và $/ \mathrm{s} /, / \mathrm{g} /$ tiếng Anh, phân biệt được các phụ âm tiếng Anh khác phát âm gần giống hai phụ âm xát $/ \mathrm{s} /, / \int /$. Sinh viên trang bị cho mình những kỹ năng tự học, tự nghiên cứu, tìm cách tiếp cận, cọ xát thực tế bằng việc học phát âm một cách có hệ thống và thường xuyên, loại bỏ tâm lý e ngại, sợ sai khi nói, luyện tập với người bản ngữ. Ngoài ra, trong quá trình học phát âm trong phòng Multimedia Lab, sinh viên cần chú ý lắng nghe cẩn thận phát âm của người bản xứ, đồng thời quan sát kĩ khẩu hình của các âm trước khi bắt đầu tập phát âm các âm đó.

\section{TÀI LIỆU THAM KHẢO}

[1] Avery, P. and Ehrlich, S. (1995), Teaching American English Pronunciation, Oxford University Press.

[2] Celce-Murcia, M., Brinton, D.M. and Goodwin, J. (1996), Teaching Pronunciation - A Reference for Teachers of English to Speakers of Other Languages, Cambridge University Press.

[3] Đoàn Thiện Thuật (1977), Sách ngữ âm tiếng việt, NXB ĐH \& THCN, Hà Nội

[4] Jenkins J. (2000), The Phonology of Engish as an International Language, Oxford University Press

[5] Mai Ngọc Chừ (1997), Cơ sở ngôn ngữ học và tiếng Việt, NXB Giáo dục, Hà Nội

[6] Mark Hancock (2003), English Pronunciation in Use, Oxford University Press

[7] Nguyễn Thiện Giáp (2008), Giáo trình ngôn ngữ học, NXB ĐHQG HN

[8] Poedjosoedarmo, G. (2004), Teaching Pronunciation - Why, What, When, and How, SEAMEO Regional Language Centre

[9] Roach, P. (2000), English Phonetics and Phonology, Cambridge University Press.

[10] Swan, M. and B. Smith (2001), Learner English (Second Edition), Cambridge University Press

Ngày nhận bài: 20/01/2018

Ngày chấp nhận đăng: 15/02/2018 